\title{
Gasto do Governo e Consumo Privado: Substitutos ou Complementares?*
}

\author{
Fábio Augusto Reis Gomes ${ }^{\dagger}$
}

\author{
Conteúdo: 1. Introdução; 2. Modelos de Consumo; 3. Evidências para o Brasil; \\ 4. Metodologia Empírica; 5. Resultados; 6. Conclusões. \\ Palavras-chave: Consumo Privado, Gasto do Governo, Substitutos, Complementares, Renda \\ Corrente. \\ Códigos JEL: $\quad$ C22, D91, E21.
}

Este artigo investiga se as alterações do consumo privado de bens não duráveis dependem do gasto do governo, levando em conta que o consumo privado pode exibir excesso de sensibilidade à renda corrente. Usando dados anuais de 1970 a 2003, conclui-se que o consumo privado não é afetado pelas mudanças previstas do gasto do governo, porém é afetado por mudanças previstas da renda. Finalmente, os resultados indicam que o consumo privado e o gasto do governo não são substitutos nem tão pouco complementares e, que há excesso de sensibilidade do consumo privado à renda.

This paper investigates whether private nondurable consumption is affected by changes in government spending, taking into account that private consumption can respond to predicted changes in current income. Using annual data from 1970 to 2003, results suggest that private consumption is not affected by anticipated changes in government spending, but it is affected by anticipated changes in income. Finally, the results indicate that private consumption and government spending are neither substitute or complementary and there is evidence of excess sensitivity to income.

\section{INTRODUÇÃO}

A literatura sobre a decisão de consumo no Brasil pode ser divida em dois grupos. O primeiro que estuda modelos lineares que relacionam mudanças no consumo à valores previstos de outras variáveis, como Reis et alii (1998), Issler e Rocha (2000), Gomes (2004, 2010), Gomes e Paz $(2004,2010)$ e o segundo

\footnotetext{
*Agradeço a Arilton Teixeira a aos pareceristas anônimos pelos comentários, isentando-os de quaisquer erros remanescentes. Agradeço ao CNPq e à FAPES pelo financiamento parcial desta pesquisa.

${ }^{\dagger}$ Fucape Business School. Av. Fernando Ferrari, 1358. Boa Vista. Vitória-ES CEP 29075-505. Tel.: (55) 27 4009-4444.

E-mail: fabiogomes@fucape.br
} 
grupo que estima a equação de Euler do consumidor diretamente, como Issler e Piqueira (2000) e Araújo (2006). ${ }^{1}$

Ao resolver o problema intertemporal do consumidor representativo pode-se obter uma equação de teste linear quando a função utilidade é quadrática ou quando se lineariza a equação de Euler obtida ao se assumir a função utilidade CRRA. Essa primeira abordagem é mais utilizada, possivelmente porque neste contexto Campbell e Mankiw (1989) propuseram uma forma de incorporar ao modelo um segundo tipo de consumidor que segue uma simples regra de bolso: consumir a renda corrente. Esse modelo híbrido é capaz de incorporar o conhecido excesso de sensibilidade do consumo a renda corrente (Flavin, 1981). ${ }^{2}$ Segundo a Teoria da Renda Permanente (TRP) o consumo deveria depender da renda permanente, porém, desde Flavin (1981) diversos autores documentaram que o consumo responde excessivamente a renda corrente. De fato, espera-se que a parte previsível da renda corrente não afete o consumo, porém os resultados de Campbell e Mankiw $(1989,1990)$ mostraram o contrário.

Segundo Flavin (1985) o excesso de sensibilidade do consumo a renda se deve à restrição de crédito, pois neste caso o consumidor não está apto a suavizar seu consumo, estando fadado a depender somente da renda corrente. Estudos mais recentes também indicam que a restrição de crédito é um fator importante (Vaidyanathan, 1993, Engelhardt, 1996, Garcia et alii, 1997, Sarno e Taylor, 1998, Brady, 2008). Outras explicações para o excesso de sensibilidade do consumo à renda incluem: poupança precaucional (Carrol, 1997, Madsen e McAleer, 2000, Gourinchas e Parker, 2001) e problemas de self-control que surgem em modelos com desconto hiperbólico (Angeletos et alii, 2001).

Neste artigo daremos um passo adicional na literatura brasileira, investigando se o consumo privado e o consumo do governo são substitutos ou complementares, usando a abordagem proposta por Karras (1994). Para tanto, será investigado se existe correlação entre a variação do consumo privado e a variação prevista do gasto do governo. o parâmetro $\theta$ que mede esta correlação responde a questão de pesquisa deste trabalho. Conforme detalhado na Seção 2, quando $\theta>0$, o consumo privado e do governo são substitutos na medida em que aumentos no segundo diminuem a utilidade marginal do primeiro. Por outro lado, se $\theta<0$, então o consumo privado e do governo são complementares já que aumentos no gasto do governo aumentam a utilidade marginal do consumo privado.

Esta pesquisa está relacionada à importante discussão sobre o efeito crowding out do gasto do governo em outros componentes da despesa agregada, no caso o consumo privado. Por exemplo, uma estimativa positiva de $\theta$ sugere que aumentos no gasto do governo reduzem a utilidade marginal do consumo, o que eventualmente acarreta uma redução no consumo privado. Neste sentido, este artigo investiga um canal pelo qual o gasto do governo pode deslocar o consumo privado. No entanto, caso a estimativa de $\theta$ seja nula, seria prematuro concluir que não há efeito crowding out (ou crowding in), já que o artigo investiga apenas o canal via função utilidade.

Em conformidade com Karras e Evans (1996) estenderemos o modelo de Karras (1994), incorporando a possibilidade de existirem consumidores que simplesmente decidem seu consumo com base na renda corrente. Em outras palavras, será feito um modelo híbrido semelhante ao de Campbell e Mankiw (1989). Essa extensão é importante, pois a literatura sobre o Brasil tem documentado de forma exaustiva a existência de excesso de sensibilidade do consumo à renda (Reis et alii, 1998, Issler e Rocha, 2000, Gomes, 2004, Gomes e Paz, 2004).

Para conduzir a análise empírica foram utilizadas as séries de consumo de bens não duráveis, produto interno bruto e gastos do governo de 1970 a 2003, provenientes de Ellery e Teixeira (2012). ${ }^{3}$ Os resultados indicam que não há uma relação significativa entre o consumo privado e o gasto do governo. Neste sentido, o consumo do governo parece não afetar a decisão de consumo dos agentes. Por outro lado, verificou-se mais uma vez o excesso de sensibilidade do consumo à renda. Finalmente, esses re-

\footnotetext{
${ }^{1}$ Cavalcanti (1993)estimou tanto modelos lineares quanto não-lineares.

${ }^{2}$ Este modelo híbrido também pode ser estimado sem que se faça a linearização da Equação de Euler, conforme discutido por Weber (2002). No entanto, essa abordagem não é frequente, especialmente no caso brasileiro.

${ }^{3}$ Agradeço a Arilton Teixeira e Roberto Ellery por disponibilizarem a base de dados.
} 
sultados indicam que a atuação do governo terá mais efeito sobre o consumidor caso ela afete a renda corrente (no curto prazo).

O artigo está organizado do seguinte modo. Na Seção 2 apresentamos a revisão dos modelos de consumo. Na terceira seção apresentamos a revisão da literatura. Na quarta seção apresentamos os dados. A quinta seção contém os resultados. Por fim, apresentamos as conclusões.

\section{MODELOS DE CONSUMO}

Nesta seção apresentamos os modelos de consumo, com o objetivo de apresentar as equações de teste a serem estimadas. Na subseção 2.1 apresentamos o modelo básico devido a Hall (1978). A subseção 2.2 apresenta a extensão proposta por Campbell e Mankiw (1989) que contempla o excesso de sensibilidade do consumo à renda. Por fim, apresentamos os modelos propostos por Karras (1994) e Karras e Evans (1996) para testar se o consumo privado de bens não duráveis e o gasto do governo são substitutos ou complementares. O modelo proposto por Evans e Karras e Evans (1996), inclusive, leva em conta a possibilidade de o consumidor responder excessivamente à renda corrente.

\subsection{A Hipótese do Passeio Aleatório}

Hall (1978) investigou a decisão de consumo de um agente representativo forward-looking, considerando o mercado perfeitamente competitivo, sem restrição de crédito e custos de transação. $O$ agente representativo da economia maximiza o valor presente esperado de sua utilidade separável no tempo, dado por:

$$
E_{t}\left[\sum_{j=0}^{\infty} \beta^{j} u\left(C_{t+j}\right)\right], \quad(0<\beta<1)
$$

em que $E_{t}$ representa a esperança condicional às informações disponíveis no período $t$; $\beta$ é o fator de desconto intertemporal; $C_{t}$ é o consumo; e $U(\cdot)$ é a função de utilidade do agente. Tal maximização é sujeita a restrição orçamentária: $A_{t+1}=\left(A_{t}+Y_{t}-C_{t}\right) R_{t+1}$, em que a riqueza no período seguinte, $A_{t+1}$, depende da remuneração, $R_{t+1}$, da riqueza corrente, $A_{t}$, somada a poupança corrente, $S_{t} \equiv$ $Y_{t}-C_{t}$. Assim, $R_{t+1}$ representa o retorno bruto enquanto $Y_{t}$ é a renda do trabalho. A solução deste problema é caracterizada pela seguinte equação de Euler:

$$
\beta E_{t}\left[\frac{U^{\prime}\left(C_{t+1}\right)}{U^{\prime}\left(C_{t}\right)} R_{t+1}\right]=1
$$

O agente representativo escolhe quanto da sua renda é alocada para o consumo corrente e, portanto, quanto é poupado, de maneira a igualar a perda de utilidade ao abrir mão de consumo presente à utilidade que espera ganhar no período seguinte pelo consumo adicional, devidamente descontada.

Hall (1978) assumiu, ainda, três hipóteses: $i$ ) o retorno é constante, $R_{t+1}=R$, ii) tal retorno é idêntico ao recíproco do fator de desconto intertemporal, isto é, $\beta R=1$; iii) a função utilidade é quadrática. Daí resulta a hipótese do passeio aleatório do consumo:

$$
C_{t+1}=C_{t}+\varepsilon_{t+1}
$$

em que $\varepsilon_{t+1}$ é uma inovação em relação às informações disponíveis no período $t$, isto é, $E_{t}\left(\varepsilon_{t+1}\right)=0$. A equação (3) indica que ao suavizar seu consumo, o agente usa toda informação disponível, de modo que seu consumo não se altera quando a inovação é nula.

Flavin (1981) chegou à mesma conclusão ao considerar explicitamente a TRP. Segundo tal teoria, cada indivíduo consome a cada período a sua renda permanente, $Y_{t}^{P}$. Como a previsão um passo à frente da renda permanente é o seu valor corrente, isto é, $E_{t}\left(Y_{t+1}^{P}\right)=Y_{t}^{P}$, é obtida dessa forma a hipótese do 
passeio aleatório do consumo. Neste sentido, os trabalhos de Hall (1978) e Flavin (1981) recuperam a contribuição de Friedman (1957), compatibilizando-a com o problema do consumidor representativo.

\subsection{Excesso de Sensibilidade do Consumo à Renda}

Flavin (1981) analisou como alterações na renda corrente afetam a renda permanente e, por conseguinte, o consumo, concluindo que o consumo reage excessivamente às variações da renda corrente. Em outras palavras, Flavin (1981) encontrou evidências de que a variação do consumo é superior a variação da renda permanente, induzida pela variação inicial da renda. Daí o termo excesso de sensibilidado do consumo à renda. ${ }^{4}$

Campbell e Mankiw (1989) propuseram um modelo híbrido no qual uma fração dos consumidores suaviza o consumo enquanto os demais seguem uma simples regra de bolso: consumir a renda corrente. Este modelo foi, diversas vezes, aplicado ao Brasil e os resultados indicam que não se pode rejeitar a hipótese de que parte dos consumidores tem seu consumo determinado pela renda corrente. ${ }^{5}$ Por essa razão, antes de introduzirmos o gasto do governo no problema apresentamos o modelo de Campbell e Mankiw (1989).

Quando a renda corrente é o determinante exclusivo do consumo, as variações da renda impactam diretamente as variações do consumo. Por outro lado, se o agente suaviza seu consumo, como descrito na subseção 2.1, então a variação do consumo é imprevisível. Diante disso, para estimar a proporção de consumidores em cada grupo, Campbell e Mankiw (1989) construíram a seguinte equação de teste: ${ }^{6}$

$$
\Delta C_{t+1}=\tilde{\mu}+\lambda \Delta Y_{t+1}+\tilde{\varepsilon}_{t+1}
$$

Tal equação pode ser vista como uma forma de aninhar dois modelos distintos. Mais precisamente, considere a seguinte equação:

$$
\Delta C_{t+1}=\lambda \Delta Y_{t+1}+(1-\lambda)\left(\mu+\zeta_{t+1}\right)
$$

Quando $\lambda=1$ temos apenas consumidores que consomem a renda corrente. Por outro lado, se $\lambda=$ 0 , voltamos ao passeio aleatório. Note que, por essa perspectiva, $\tilde{\mu}=(1-\lambda) \mu$ e $\tilde{\varepsilon}_{t+1}=(1-\lambda) \varepsilon_{t+1}$. Finalmente, quando $\lambda$ é significativo, diz-se que existe excesso de sensibilidade do consumo à renda. Como mencionado, esse termo foi usado inicialmente por Flavin (1981) para documentar um impacto excessivo da renda corrente sobre o consumo nos Estados Unidos.

Devido à endogeneidade da taxa de juros, na prática os modelos são estimados por meio de variáveis instrumentais. Por exemplo, aplicando o estimador de mínimos quadrados em dois estágios (MQ2E), no primeiro estágio é feita a regressão das variáveis endógenas nos instrumentos, que geralmente são variáveis defasadas. Portanto, é construído o valor previsto da variação da renda, $\widehat{\Delta Y}_{t+1}$. Note que, além de econometricamente correto, esse procedimento está de acordo com a TRP. Se fosse usada a renda diretamente, então $\lambda$ seria diferente de zero, simplesmente porque a inovação da renda corrente contém informação sobre mudanças na renda permanente. Portanto, estima-se, na prática, o efeito da variação esperada da renda sobre a variação do consumo.

A literatura aponta vários motivos para $\lambda$ ser diferente de zero. O predominante é a existência de restrição de crédito, conforme discutido, por exemplo, por Vaidyanathan (1993), Engelhardt (1996),

\footnotetext{
${ }^{4}$ Flavin (1985) argumentou que tal resultado se deve a falta de acesso ao crédito, pois nesta situação o consumo torna-se extremamente dependente da renda corrente.

${ }^{5}$ Na Seção 3 são detalhadas as aplicações deste modelo ao caso brasileiro.

${ }^{6}$ Campbell e Mankiw (1989) consideraram também uma equação de teste na qual é utilizada o logaritmo das séries. Essa equação é obtida quando se assume uma função CRRA e a hipótese de log-normalidade conjunta do consumo e do retorno. A vantagem desse modelo é que o retorno passa a fazer parte da equação de teste. No entanto, sempre que tal versão é aplicada ao caso brasileiro, o retorno não se mostra relevante. Por essa razão, optamos pela versão sem o retorno, que foi também utilizada por Karras e Evans (1996), conforme descrito na subseção 2.3.
} 
Garcia et alii (1997), Sarno e Taylor (1998) e Brady (2008). Por ouro lado, Carrol (1997) advoga que o modelo de poupança precaucional é capaz de explicar por que $\lambda$ é diferente de zero. ${ }^{7}$ A ideia básica é que o consumidor tem uma meta de riqueza em relação à renda permanente e uma vez atingida essa meta o indivíduo consume sua renda corrente. Além disso, Angeletos et alii (2001) argumentam que problemas de self-control que surgem em modelos com desconto hiperbólico também podem explicar porque $\lambda$ é diferente de zero.

\subsection{Gasto do Governo}

Karras (1994) e Karras e Evans (1996) analisaram se o consumo do governo é substituto ou complementar do consumo privado. Inicialmente, os autores supuseram que os consumidores se beneficiam tanto com o gasto privado quanto com o gasto do governo. Assim, em conformidade com Bailey (1971), o consumo efetivo, $C_{t}^{*}$, foi definido como

$$
C_{t}^{*}=C_{t}+\theta g_{t}
$$

em que $C_{t}$ é, como antes, o consumo privado no período $t, g_{t}$ é o gasto do governo no período $t$ e $\theta$ é o parâmetro que mede o grau de substituição ou complementariedade entre o consumo privado e do governo. Assim, o problema do consumidor representativo torna-se maximizar

$$
E_{t}\left[\sum_{j=0}^{\infty} \beta^{j} U\left(C_{t+j}^{*}\right)\right]
$$

Esta maximização é sujeita a restrição orçamentária. Além disso, note que, quando $\theta>0, C_{t}$ e $g_{t}$ são substitutos na medida em que aumentos em $g_{t}$ diminuem a utilidade marginal de $C_{t}$. Esse efeito é tão maior, quanto maior $\theta$. Por outro lado, se $\theta<0$, então $C_{t}$ e $g_{t}$ são complementares já que aumentos em $g_{t}$ aumentam a utilidade marginal de $C_{t}$.

Procedendo de maneira análoga a Hall (1978), é obtida a hipótese do passeio aleatório para o consumo efetivo:

$$
C_{t+1}^{*}=C_{t}^{*}+\zeta_{t+1}
$$

Portanto, a variação do consumo efetivo é imprevisível: $\Delta C_{t+1}^{*}=\zeta_{t+1}$. Como $C_{t}^{*}=C_{t}+\theta g_{t}$, fica evidente que

$$
\Delta C_{t+1}=-\theta \Delta g_{t}+\zeta_{t+1}
$$

Este modelo foi estimado por Karras (1994), usando dados de 30 países e os resultados sugerem que o consumo privado e público são descritos mais adequadamente como complementares ou independentes. Karras e Evans (1996) estenderam esse modelo com o intuito de contemplar o modelo híbrido de Campbell e Mankiw (1989). Para tanto, assumiu-se que parte dos consumidores se comporta de acordo com a equação (9), enquanto os demais seguem a regra de bolso de consumir a renda corrente. Foi construída, então, a seguinte equação de teste:

$$
\Delta C_{t+1}=\tilde{\mu}+\lambda \Delta Y_{t+1}-\tilde{\theta} \Delta g_{t}+\tilde{\zeta}_{t+1}
$$

De maneira semelhante ao modelo de Campbell e Mankiw (1989), tal equação pode ser vista como uma forma de aninhar dois modelos distintos. Mais precisamente, considere a seguinte equação:

$$
\Delta C_{t+1}=\lambda \Delta Y_{t+1}+(1-\lambda)\left(\mu-\theta \Delta g_{t}+\zeta_{t+1}\right)
$$

\footnotetext{
${ }^{7}$ Ver, também, Madsen e McAleer (2000) e Gourinchas e Parker (2001).
} 
Quando $\lambda=1$ temos apenas consumidores que consomem a renda corrente. Por outro lado, se $\lambda=0$, voltamos à equação (9). Por essa perspectiva, $\tilde{\mu}=(1-\lambda) \mu, \tilde{\theta}=(1-\lambda) \theta$ e $\tilde{\zeta}_{t+1}=(1-\lambda) \zeta_{t+1}$. Como $0<\lambda<1$, então $\tilde{\theta}$ tem o mesmo sinal de $\theta$, podendo ser feita imediatamente a distinção entre o caso de substituição e complementariedade no consumo privado e público. Conforme mencionado, a estimação é feita por meio de variáveis instrumentais e, por conseguinte, $\lambda$ mede o impacto da variação esperada da renda sobre o consumo enquanto $(1-\lambda) \theta$ mede o impacto da variação prevista do gasto do governo sobre o consumo.

Usando dados de 54 países Karras e Evans (1996) estimaram a equação (11) impondo $\lambda=0$, ou seja, estimaram o modelo de Karras (1994). Os resultados indicam que o consumo do governo e o consumo privado parecem ser complementares. Ao estimar o modelo (11) completo, foi enfraquecida a evidência de complementariedade, pois as estimativas de $\theta$ deslocaram-se na direção de valores positivos. Além disso, a maioria dos países mostrou sinal de excesso de sensibilidade do consumo à renda.

\section{EVIDÊNCIAS PARA O BRASIL}

Nesta seção revisamos a literatura nacional que trata da aplicação do modelo de Campbell e Mankiw (1989), ao invés de fazer uma revisão exaustiva da literatura. Essa opção foi feita porque é investigado se os gastos do governo estão relacionados a mudanças do consumo privado, levando-se em conta que o consumidor pode reagir excessivamente às mudanças da renda corrente. Além disso, nos limitamos a tal parte da literatura, pois não se tem conhecimento de outros artigos que investigam tal impacto do gasto do governo sobre o consumidor.

Um dos primeiros estudos da literatura nacional sobre teoria de consumo é o de Cavalcanti (1993), cujo objetivo era testar as implicações da TRP e investigar a existência de excesso de sensibilidade do consumo à renda. Utilizando dados trimestrais do consumo agregado per capita e do PIB per capita, de 1980 a 1989, encontrou-se evidências de não estacionariedade nas séries e, posteriormente, estimou $\lambda$ aproximadamente igual a 0,32. Não se rejeita, portanto, a existência de excesso de sensibilidade do consumo à renda. Caso a causa desse fenômeno seja, exclusivamente, a existência de restrição de crédito, então, pode-se dizer que $32 \%$ da renda no Brasil, no período analisado, pertencia a consumidores restritos ao crédito.

Reis et alii (1998) estudaram várias séries anuais de consumo e de renda per capita, no período de 1947 a 1994, além de séries trimestrais de janeiro de 1975 a abril de 1994. Conforme predito pela TRP, os resultados obtidos sugerem que as séries de renda e consumo são cointegradas. Além disso, o valor mediano das estimativas de $\lambda$ é igual a 0,8. Caso o motivo desse excesso de sensibilidade a renda seja, unicamente, a restrição de crédito, isso significa que aproximadamente $80 \%$ da renda no Brasil pertence a consumidores que estão restritos a consumir apenas a sua renda corrente, estimativa bastante superior à encontrada por Cavalcanti (1993). Uma possível explicação para essa diferença é o uso de instrumentos diferentes na aplicação do método de variáveis instrumentais. Porém, outra possibilidade é a não-estabilidade temporal de $\lambda$, dado que Cavalcanti (1993) e Reis et alii (1998) estudaram períodos diferentes. Posteriormente, os resultados de Issler e Rocha (2000) corroboraram as conclusões de Reis et alii (1998), pois estimou-se $\lambda$, aproximadamente, igual a 0,74, para o período de 1947 a 1994.

Gomes (2004) encontrou evidências de que o consumo agregado, em frequência anual de 1947 a 1999, possui um componente cíclico, o que vai de encontro a TRP. Esse componente cíclico indica que o consumo não segue um passeio aleatório. Em uma tentativa de explicar este resultado, Gomes (2004) considerou duas alternativas: formação de hábito e excesso de sensibilidade do consumo à renda. Os resultados dão suporte à segunda explicação, pois ao estimar o modelo de Campbel e Mankiw (1989), acrescido de uma defasagem do consumo devido à formação de hábito, concluiu-se que $\lambda$ é igual a 0,85 e não houve evidência favorável a hipótese de formação de hábito.

Gomes e Paz (2004) analisaram a evolução do consumo em 7 países da América do Sul (Argentina, Brasil, Chile, Colômbia, Peru, Paraguai e Uruguai), por meio do modelo de Campbell e Mankiw (1989). 
A Argentina e o Chile foram os países que apresentaram as maiores estimativas de $\lambda$ ao passo que o Peru foi o país que apresentou a menor estimativa. O Brasil ocupou uma posição intermediária com $\lambda$, aproximadamente, igual a 0,61. De todo modo, esse resultado indica um claro excesso de sensibilidade do consumo à renda no Brasil.

A maior parte da literatura brasileira testa as implicações de modelos de consumo para bens não duráveis por meio de séries de consumo que incluem bens duráveis e não duráveis. Mas, as implicações comumente testadas são obtidas ao se modelar separadamente a decisão de consumo de bens duráveis e de bens não duráveis. Nesse sentido, a validade dos estudos anteriores depende de uma hipótese de separabilidade entre duráveis e não duráveis. Gomes et alii (2005) analisaram essa hipótese de separabilidade com base nas séries construídas por Ellery Jr. et alii (2002) para os dois tipos de gastos. Os autores não rejeitaram a hipótese de separabilidade e concluíram que há forte evidência de excesso de sensibilidade do consumo, tanto de bens duráveis quanto de não duráveis, à renda. Esses resultados foram interpretados como uma evidência de restrição de crédito.

Portanto, há forte evidência de que $\lambda$ é diferente de zero no Brasil. Isso implica que para estudarmos o efeito do gasto do governo sobre o consumo não podemos nos limitar ao modelo de Karras (1994), sendo necessário considerar a versão proposta por Karras e Evans (1996).

\section{METODOLOGIA EMPÍRICA}

\subsection{Base de dados}

Para estimar as equações (4), (9) e (10) é necessário ter informações sobre o consumo privado de bens não duráveis e os gastos do governo. O IBGE calcula o consumo final das famílias, agregando o consumo de bens duráveis e não duráveis. Como mencionado, em geral, os estudos têm analisado as propriedades de tal série, usando os modelos concebidos para a decisão de consumo de bens não duráveis (Reis et alii, 1998, Issler e Rocha, 2000, Gomes, 2004, Gomes e Paz, 2004). As exceções são Gomes et alii (2005) e Gomes (2013) que trabalharam de forma distinta o consumo de bens duráveis e não duráveis. Para tanto, Gomes et alii (2005) usaram as séries de consumo de duráveis e de não duráveis construídas por Ellery Jr. et alii (2002) referentes ao período de 1970 a 1998, enquanto Gomes (2013) utilizou tais séries estendidas até o ano de 2003, provenientes de Ellery e Teixeira (2012). Usaremos esta última base de dados.

Gomes et alii (2005) tinham como foco principal testar se a função utilidade do consumidor representativo é separável nos dois tipos de bens. Em caso afirmativo, isso permite que o consumo de bens duráveis e não duráveis sejam estudados separadamente. Nesse sentido, tal análise é fundamental para validar os estudos anteriores e, de fato, como mencionado, Gomes et alii (2005) não rejeitaram a hipótese de separabilidade. Gomes (2013) tinha como foco investigar possíveis distinções na evolução dos gastos com bens duráveis e não duráveis. As evidências indicaram que as duas séries tem comportamento distinto, sendo preferível estudá-las separadamente, ao invés de usar a série de consumo composta pelos dois tipos de bens.

Diferentemente desses artigos, queremos investigar a relação entre o consumo de bens não duráveis e o gasto do governo, mas para tanto nos apoiaremos no teste de separabilidade feito por Gomes et alii (2005), estudando somente a evolução e os determinantes do consumo de bens não duráveis.

Os dados de consumo de não duráveis, consumo do governo e renda em termos reais foram divididos pela população residente no Brasil, calculada pelo IBGE, de modo a serem construídas séries reais per capita. A evolução do produto interno bruto (PIB), consumo de bens não duráveis (CND) e gastos do governo (CG) é apresentada na Figura 1. Notamos que o crescimento acentuado do inicio da década de 70 é sucedido por uma redução (em termos reais) do PIB per capita na década de 80, a chamada década perdida. Em seguida ocorreu uma estagnação do PIB, com uma aceleração por volta de 1994, ano no qual foi implementado o Plano Real de estabilização. Mas, ainda na década de 90 ocorreu uma ligeira queda do PIB real per capita. O consumo de não duráveis não apresentou uma queda, pelo menos não 
tão pronunciada como a do PIB, no início da década de 80. Porém, ao final desta década houve uma queda considerável. Mas, de um modo geral, a evolução do consumo tem semelhanças com a do PIB. Já o gasto do governo, parece ser quase sempre crescente, independentemente do comportamento do PIB.

Figura 1: Evolução do Produto Interno Bruto, Consumo de $\mathrm{N}^{0}$ Duráveis e Gastos do Governo

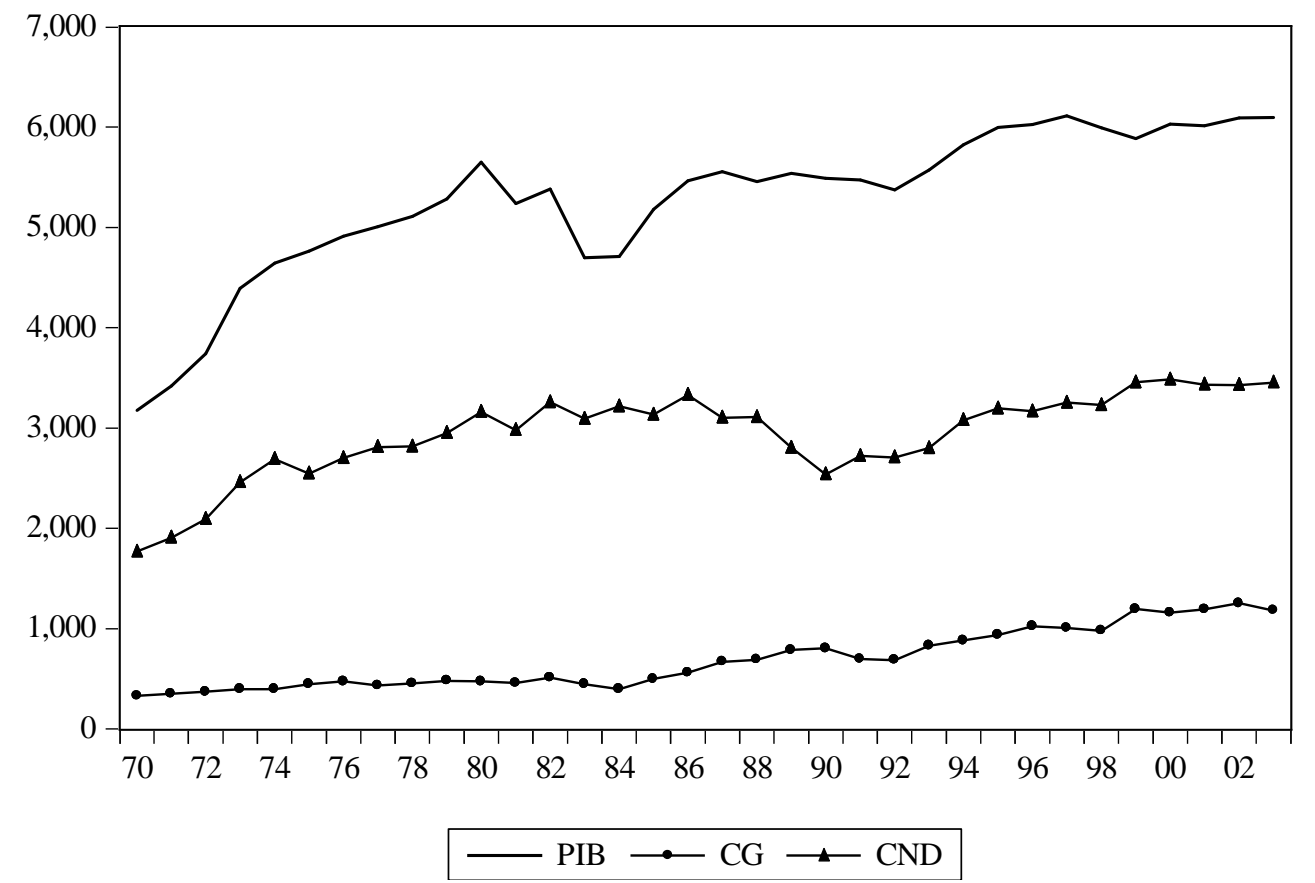

Foi feita, então, a análise da ordem de integração das séries por meio de testes de raiz unitária. Foram utilizados os testes ADF, PP e ADF-GLS cuja hipótese nula é a existência de raiz unitária (nãoestacionariedade), além do teste KPSS cuja hipótese nula é estacionariedade. ${ }^{8}$ Aplicamos tais testes às séries em nível e primeira diferença. Quando em nível foram consideradas duas especificações para os termos determinísticos:

i) constante;

ii) constante e tendência linear.

Quando em primeira diferença, apenas a constante foi incluída.

Os resultados dos testes de raiz unitária são apresentados na Tabela 1. Com relação ao PIB os resultados são mistos. Os testes ADF e PP rejeitam a hipótese nula de raiz unitária, enquanto o teste ADF-GLS chega ao resultado oposto. $O$ teste KPSS também sugere que o PIB não é estacionário. Quanto ao consumo de não duráveis, os resultados dependem do componente determinístico. Se incluída apenas a constante, a 5\% de significância, todos os testes indicam que a série é I(1). Ao incluir a tendência linear, o KPSS deixa de rejeitar a hipótese nula de estacionariedade. De todo modo, em geral, há uma indicação de raiz unitária. No caso do gasto do governo, todos os testes indicam que há raiz unitária, a $5 \%$ de significância. Por fim, quando diferenciadas, todas as séries parecem ser $\mathrm{I}(0)$, sendo este o resultado indicado por todos os testes a qualquer nível usual de significância.

\footnotetext{
${ }^{8}$ Para maiores detalhes ver Dickey e Fuller (1979), Phillips e Perron (1988), Elliott et alii (1996) e Kwiatkowski et alii (1992).
} 
Tabela 1: Testes de Raiz Unitária

\begin{tabular}{lccccc}
\hline \multicolumn{1}{c}{ Série } & Termos Determinísticos & ADF & PP & ADF-GLS & KPSS \\
\hline PIB & Constante & $-3.186^{* *}$ & $-3.637^{* *}$ & $-0,745$ & $0,708^{* *}$ \\
PIB & Const., tend. linear & $-4.530^{*}$ & $-3.924^{* *}$ & -2.196 & $0,123^{* * *}$ \\
CND & Constante & $-2.755^{* * *}$ & $-2.770^{* * *}$ & $-0,829$ & $0,533^{* *}$ \\
CND & Const., tend. linear & -2.610 & -2.613 & -1.973 & 0,116 \\
CG & Constante & $-0,091$ & $-0,015$ & 0,430 & $0,646^{* *}$ \\
CG & Const., tend. linear & -2.238 & -2.144 & -2.165 & $0,182^{* *}$ \\
$\Delta$ PIB & Constante & $-4.765^{*}$ & $-4.709^{*}$ & $-4.671^{*}$ & 0,294 \\
$\Delta$ CND & Constante & $-5.864^{*}$ & $-5.861^{*}$ & $-3.014^{*}$ & 0,239 \\
$\Delta$ CG & Constante & $-6.019^{*}$ & $-6,019^{*}$ & $-5.965^{*}$ & 0,112 \\
\hline
\end{tabular}

Nota: ${ }^{*},{ }^{* *},{ }^{* *}$ indicam rejeições da hipótese nula a $1 \%, 5 \%$ e $10 \%$, respectivamente. $\mathrm{O}$ número de defasagens da variável dependente incluídas na equação de teste $\mathrm{ADF}$ foi escolhido com base no critério de informação de Schwarz.O teste de PP usou o núcleo de Bartlett e a janela de Newey-West.

A Figura 2 apresenta a evolução da variação do consumo de bens não duráveis, do gasto do governo e do PIB. Visualmente, as variações do consumo parecem ser mais semelhantes às variações do PIB do que as do gasto do governo. De fato, a correlação entre as variações do consumo privado e do PIB é, aproximadamente, 0,53 , sendo significativa a $1 \%$. Já a correlação da variação do consumo privado e do governo é, aproximadamente, $-0,02$, não sendo significativa, mesmo a $10 \%$ de significância. Por fim, note que o consumo de não duráveis oscila consideravelmente; no entanto, é importante levar em conta que a média de tal série é bastante superior a de gastos do governo.

Como a primeira diferença das séries não apresenta raiz unitária, apresentamos na Tabela 2 as estatísticas descritivas de tais séries. A maior variação média é a do PIB. Como as séries apresentam médias distintas, analisamos o coeficiente de variação como medida de dispersão. Em relação à média, a maior variabilidade é a do gasto do governo, superior inclusive a do PIB. O consumo tem a menor variabilidade, em relação à média. Em termos de amplitude, o consumo também apresenta o menor valor. Portanto, por essas duas medidas de variabilidade, o consumo é a série mais suave.

\subsection{Modelo Econométrico}

Nesta subseção discutimos como o MGM pode ser aplicado para estimar os parâmetros de interesse.

Seja $h\left(Z_{t} ; \theta\right)$ um modelo com r equações, no qual $Z_{t}$ é um vetor $1 \times k$ de $k$ variáveis e $\theta$ é um vetor de parâmetros $a \times 1$. Assuma que o número de equações é igual ou superior ao número de parâmetros, isto é, $r \geq a$. Suponha que as variáveis contidas em $Z_{t}$ sejam integradas de ordem zero e que exista um único valor de $\theta$, digamos $\theta_{0}$, tal que $E\left[h\left(Z_{t} ; \theta_{0}\right)\right]=0$. Neste caso, o MGM pode ser aplicado para estimar o vetor de parâmetros $\theta_{0}$. O método consiste, basicamente, na substituição do momento populacional, $E\left[h\left(Z_{t} ; \theta\right)\right]$, pelo momento amostral que é dado por:

$$
g\left(\left\{Z_{t}\right\}_{t=1}^{T} ; \theta\right)=\frac{1}{T} \sum_{t=1}^{T} h\left(Z_{t} ; \theta\right)
$$


Figura 2: Variação do Produto Interno Bruto, Consumo de $\mathrm{N}^{\circ}$ Duráveis e Gastos do Governo

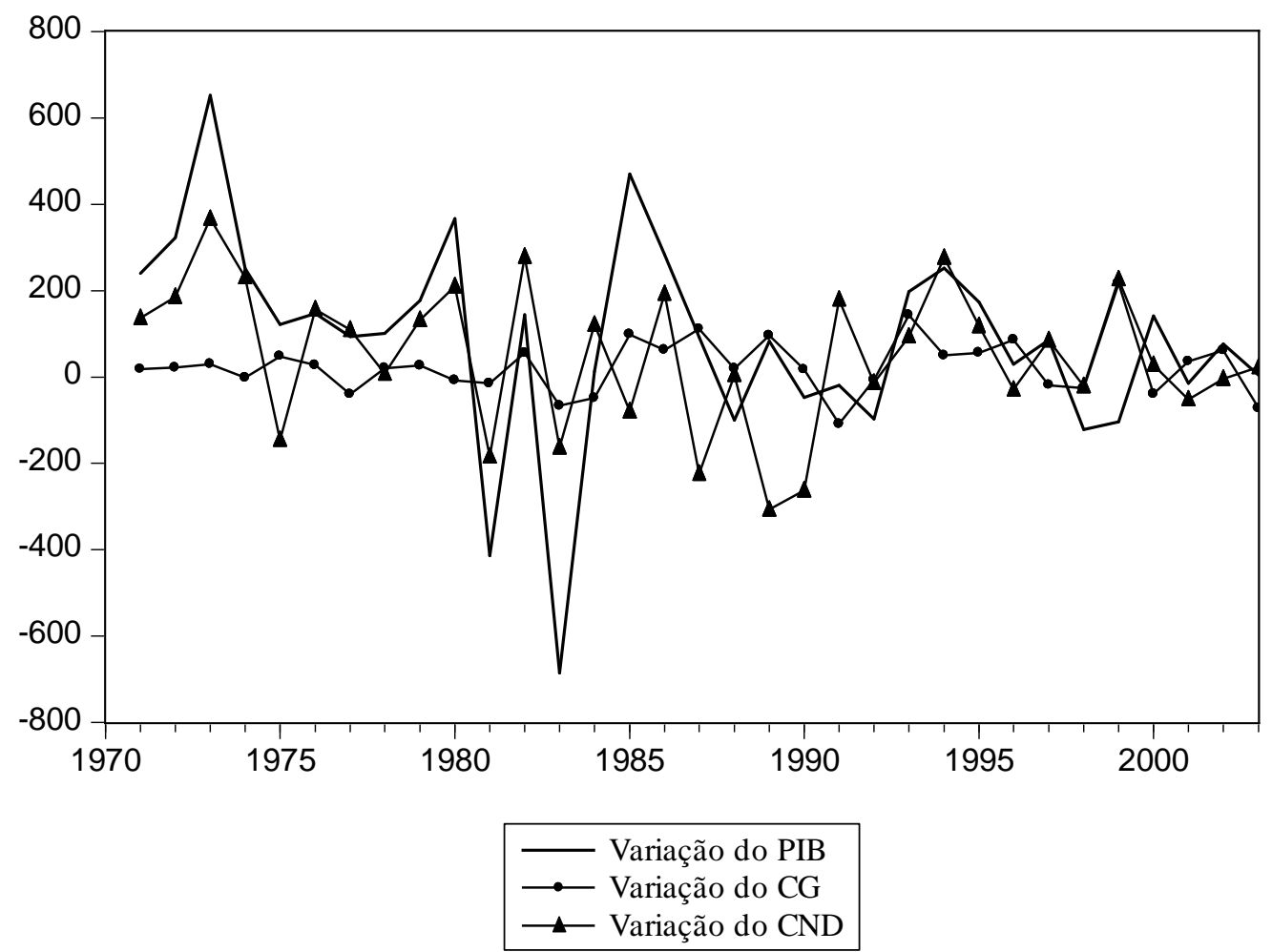

Tabela 2: Estatística Descritiva

\begin{tabular}{lccc}
\hline \multicolumn{1}{c}{ Estatística } & $\Delta \mathrm{PIB}$ & $\Delta \mathrm{CND}$ & $\Delta \mathrm{CG}$ \\
\hline Média & 88.419 & 25.833 & 50.970 \\
Desvio-Padrão & 237.810 & 65.446 & 165.769 \\
Coeficiente de Variação & 2.690 & 2.533 & 3.252 \\
Máximo & 652.728 & 216.581 & 366.396 \\
Mínimo & -685.553 & -108.505 & -306.706 \\
Amplitude & 1.338 .281 & 325.086 & 673.102 \\
\hline
\end{tabular}

Nota: $\Delta$ é o operador de primeira diferença. 
No tradicional método dos momentos o número de parâmetros é igual ao número de condições de momento, bastando igualar os momentos populacionais aos respectivos momentos amostrais para se obter uma estimativa para cada parâmetro. O MGM permite que o número de condições de momento seja superior ao número de parâmetros, $r>a$. Neste caso, o estimador do MGM é obtido ao se minimizar uma forma quadrática dos momentos amostrais, isto é,

$$
\hat{\theta}_{M G M}=\operatorname{argmin}\left\{g\left(\left\{Z_{t}\right\}_{t=1}^{T} ; \theta\right)^{\prime} W g\left(\left\{Z_{t}\right\}_{t=1}^{T} ; \theta\right)\right\}
$$

em que $W$ é uma matriz positiva definida. É possível mostrar que a escolha ótima para essa matriz é o inverso da variância assintótica do momento amostral. ${ }^{9}$

A hipótese de expectativas racionais implica que o termo de erro das equações (4), (9) e (10), não são correlacionados com variáveis defasadas, assim, podemos escrevê-las do seguinte modo: ${ }^{10}$

$$
\begin{gathered}
E_{t}\left[\Delta C_{t+1}-\tilde{\mu}-\lambda \Delta Y_{t+1}\right]=0 \\
E_{t}\left[\Delta C_{t+1}-\mu+\theta \Delta g_{t}\right]=0 \\
E_{t}\left[\Delta C_{t+1}-\tilde{\mu}-\lambda \Delta Y_{t+1}+\tilde{\theta} \Delta g_{t}\right]=0
\end{gathered}
$$

Portanto, como cada condição de momento é proveniente de um modelo com expectativas racionais, cada uma deve ser ortogonal a qualquer variável que pertence ao conjunto de informação do consumidor, $I_{t}$. Seja $X_{t} \subset I_{t}$ tal que $X_{t} \equiv\left[\begin{array}{llll}x_{t} & x_{t-1} & \cdots & x_{t-j}\end{array}\right]$. Note que j é o número de defasagens incluídas em $X_{t}$. Então, as condições de momento podem ser ampliadas:

$$
\begin{gathered}
E_{t}\left[\left(\Delta C_{t+1}-\tilde{\mu}-\lambda \Delta Y_{t+1}\right) \otimes X_{t}\right]=0 \\
E_{t}\left[\left(\Delta C_{t+1}-\mu+\theta \Delta g_{t}\right) \otimes X_{t}\right]=0 \\
E_{t}\left[\left(\Delta C_{t+1}-\tilde{\mu}-\lambda \Delta Y_{t+1}+\tilde{\theta} \Delta g_{t}\right) \otimes X_{t}\right]=0
\end{gathered}
$$

Nos dois primeiros casos temos 2 parâmetros e $j$ condições de momento, bastando que $j$ seja igual ou superior a 2 . No terceiro caso como existem 3 parâmetros, $j$ deve ser pelo menos igual a 3. Por fim, usando a lei das expectativas iterada, obtemos momentos incondicionais:

$$
\begin{gathered}
E\left[\left(\Delta C_{t+1}-\tilde{\mu}-\lambda \Delta Y_{t+1}\right) \otimes X_{t}\right]=0 \\
E\left[\left(\Delta C_{t+1}-\mu+\theta \Delta g_{t}\right) \otimes X_{t}\right]=0 \\
E\left[\left(\Delta C_{t+1}-\tilde{\mu}-\lambda \Delta Y_{t+1}+\tilde{\theta} \Delta g_{t}\right) \otimes X_{t}\right]=0
\end{gathered}
$$

Assim, a aplicação do MGM é imediata. Quando o número de condições de momento é superior ao número de parâmetros, podemos aplicar o teste $J$ de sobreidentificação, cuja hipótese nula é a validade das condições de momento. Nesta perspectiva, esse teste investiga a validade do modelo utilizado.

O estimador de MQ2E, bastante usual na literatura de consumo, é um caso particular do MGM. De fato, em modelos lineares e homocedásticos o MGM e o MQ2E coincidem. A vantagem do MGM é que

\footnotetext{
${ }^{9}$ Para maiores detalhes sobre o método de minimização da função objetivo do MGM bem como sobre a matriz W, ver Hamilton (1994).

${ }^{10}$ Foi incluída uma constante na equação (9).
} 
a matriz de peso pode ser estimada de maneira robusta a heterocedasticiadade e a correlação serial, o que influencia tanto a estimativa dos erros-padrão quanto a dos parâmetros (quando $r>a$ ). De todo modo, a relação com o MQ2E nos ajuda a entender como o modelo de consumo é testado. No primeiro estágio do MQ2E é feita a regressão das variáveis endógenas nos instrumentos. Como os instrumentos são variáveis defasadas, na prática estão sendo construídas previsões da variação da renda e do gasto do governo. Assim, no segundo estágio é feita a regressão da variação do consumo em relação às mudanças previsíveis da renda e do gasto do governo.

A inovação da renda tem informação sobre a renda permanente e, portanto, o fato de a renda impactar o consumo não contradiz a TRP. No entanto, a previsão da renda não contém informação sobre a inovação da renda permanente, não devendo, portanto impactar o consumo, caso a TRP seja valida. Além da endogeneidade, isso explica porque usamos um método de variáveis instrumentais. Queremos avaliar se mudanças previsíveis da renda e do gasto do governo levam a ajustes do consumo.

\section{RESULTADOS}

Nesta seção apresentamos a estimação dos seguintes modelos:

i) Hall (1978), passeio aleatório do consumo;

ii) Campbell e Mankiw (1989), modelo híbrido que mede o excesso de sensibilidade do consumo à renda, descrito pela equação (4);

iii) Karras (1994), modelo com gasto de governo, descrito pela equação (9);

iv) Evans e Karras e Evans (1996), modelo híbrido com gasto do governo, descrito pela equação (10).

O modelo de Hall (1978) que possui apenas uma constante foi estimado pelo método de Mínimos Quadrados Ordinários (MQO). Os demais modelos foram estimados pelo MGM, sendo considerados 4 conjuntos distintos de instrumentos. Os instrumentos são sempre as defasagens das variáveis que aparecem na equação de teste. 0 primeiro conjunto de instrumentos usa da primeira a terceira defasagens. O segundo conjunto usa da segunda a quarta defasagens. O terceiro conjunto usa apenas a primeira e a segunda defasagens. Por fim, o quarto conjunto usa a segunda e a terceira defasagens. São considerados diversos conjuntos com o intuito de verificar se os resultados são robustos. Além disso, quanto maior o número de instrumentos, menor o poder do teste $\mathrm{J}$ e, por essa, razão, usamos os dois últimos conjuntos com menos instrumentos. Os resultados são apresentados na Tabela 3.

Inicialmente, estimamos o passeio aleatório e, portanto, o modelo para a variação do consumo inclui apenas a constante, cujo $p$-valor foi superior a 10\%. Estimamos, então, o modelo de Campbell e Mankiw (1989) que inclui a renda, isto é, a equação (4). Em três casos o parâmetro $\lambda$ foi estatisticamente diferente de zero, a $5 \%$ de significância. Nestes três casos o valor médio de $\hat{\lambda}$ é, aproximadamente, 0,668 , indicando um elevado grau de dependência do consumo em relação à renda prevista. Em todos os conjuntos de instrumentos o teste $\mathrm{J}$ não rejeitou a validade das condições de momento, a $5 \%$ de significância. Portanto, de um modo geral, não se rejeita que existe um excesso de sensibilidade do consumo a renda. Em outras palavras, existe evidência de que o consumidor altera seu consumo quando ocorre uma variação prevista na renda. Esses resultados estão de acordo com os estudos apresentados na seção 3.

Os resultados para o modelo de Karras (1994), que inclui apenas as mudanç as no gasto do governo, são também apresentados na Tabela 3. A estimativa pontual de $\theta$ é instável, porém, não foi significativa em nenhum conjunto de instrumento, a 5\% de significância. Nesta perspectiva, a variação (prevista) do consumo do governo parece não causar qualquer mudança no consumo privado. É importante destacar que em todos os conjuntos de instrumentos, o teste $\mathrm{J}$ não rejeitou a validade das condições de momento, a 5\% de significância. 
Tabela 3: Estimação pelo MGM de modelo para $\Delta$ CND

\begin{tabular}{|c|c|c|c|c|c|}
\hline Modelo & $\begin{array}{c}\text { Defasagens } \\
\text { dos Instrumentos }\end{array}$ & Constante & $\Delta \mathrm{PIB}$ & $-\Delta C G$ & Teste $J$ \\
\hline \multirow{10}{*}{ Campbell e Mankiw } & & 50970 & & & \\
\hline & & $(0,108)$ & & & - \\
\hline & & $-8,002$ & $0,689^{*}$ & & 1,903 \\
\hline & $1^{\omega}, 2^{\omega}$ e $3^{\omega}$ & $(0,709)$ & $(0,000)$ & & $(0,862)$ \\
\hline & $a>a$ & 6,748 & $0,676^{*}$ & & 2,847 \\
\hline & $2^{\omega}$, & $(0,797)$ & $(0,000)$ & & $(0,723)$ \\
\hline & $1^{a} \boldsymbol{2}^{a}$ & 16,023 & 0,423 & & 0,943 \\
\hline & & $(0,751)$ & $(0,315)$ & & $(0,815)$ \\
\hline & $2^{a} 2^{a}$ & 12,623 & $0,637^{*}$ & & 0,708 \\
\hline & & $(0,666)$ & $(0,006)$ & & $(0,871)$ \\
\hline \multirow{8}{*}{ Karras } & $1^{a} \quad 2^{a} \quad 3^{a}$ & $63,578^{* *}$ & & 0,361 & 5,166 \\
\hline & $1,2, J$ & $(0,049)$ & & $(0,583)$ & $(0,396)$ \\
\hline & $2^{a} 3^{a} \mathrm{e} 4^{a}$ & $42,221^{* *}$ & & 0,208 & 4,406 \\
\hline & & $(0,082)$ & & $(0,557)$ & $(0,493)$ \\
\hline & $1^{a}$ & 7,630 & & $-1,721$ & 1,446 \\
\hline & 1,2 & $(0,925)$ & & $(0,400)$ & $(0,695)$ \\
\hline & $2^{a} \circ 3^{a}$ & $73,436^{* *}$ & & 0,100 & 3,543 \\
\hline & 2 E & $(0,091)$ & & $(0,913)$ & $(0,315)$ \\
\hline \multirow{8}{*}{ Evans e Karras } & $1^{a}$ & 4,512 & $0,855^{*}$ & $1,349^{* *}$ & 3,800 \\
\hline & $1,2,3$ & $(0,805)$ & $(0,000)$ & $(0,037)$ & $(0,803)$ \\
\hline & $2^{a} 3^{a} \mathrm{e} 4^{a}$ & $-22,526$ & $0,915^{*}$ & 0,311 & 3,683 \\
\hline & & $(0,177)$ & $(0,000)$ & $(0,396)$ & $(0,816)$ \\
\hline & $1^{a} 2^{a}$ & $-49,544$ & $0,685^{* * *}$ & $-1,173$ & 2,425 \\
\hline & & $(0,146)$ & $(0,063)$ & $(0,430)$ & $(0,658)$ \\
\hline & $2^{a} \cap 3^{a}$ & $-8,192$ & $0,683^{*}$ & 0,094 & 3,901 \\
\hline & & $(0,775)$ & $(0,006)$ & $(0,919)$ & $(0,420)$ \\
\hline
\end{tabular}

Nota: Entre parêntesis está o $p$-valor. ***,*** indicam significância a 1\%, 5\% e 10\%, respectivamente. Foi utilizado o MGM-Iterativo para se obter as estimativas dos parâmetros. Além disso, a matriz de peso foi obtida via estimador de Newey e West (1987) com núcleo de Bartlett e a janela fixa Newey-West. 
Por fim, foi estimado o modelo de Karras e Evans (1996), isto é, a equação (10). Todas as estimativas de $\lambda$ foram estatisticamente diferentes de zero, ao nível de significância de $10 \%$. 0 valor médio de $\hat{\lambda}$ é, aproximadamente, 0,785 , superior àquele obtido no modelo (4). Quanto ao gasto do governo, as estimativas de $\tilde{\theta}$ continuam instáveis. Somente em um caso, rejeita-se que $\tilde{\theta}$ seja nulo, mesmo ao nível de significância de $10 \%$. Assim, de um modo geral, o impacto direto do valor previsto dos gastos do governo sobre o consumo privado de bens não duráveis parece ser nulo. Obviamente, caso tais gastos tenham efeito de curto prazo sobre a renda corrente, então, indiretamente o consumo é afetado. Por fim, destaca-se que, novamente, o teste J não rejeitou a validade das condições de momento, a 5\% de significância.

\section{CONCLUSÕES}

Este artigo investigou se o consumo privado e o gasto do governo são substitutos ou complementares, levando em conta que o consumidor brasileiro pode ser excessivamente sensível à renda corrente, conforme amplamente discutido na literatura nacional. Para realizar essa análise foi utilizada uma série de consumo de bens não duráveis, ao invés da usual série composta por bem duráveis e não duráveis. A investigação sobre o impacto do gasto do governo no consumo privado, bem como o uso de uma série de consumo de bens não duráveis constituem as principais contribuições deste trabalho.

Os resultados sugerem que existe excesso de sensibilidade do consumo a renda e, neste sentido, a renda corrente tem um papel primordial na determinação do consumo. A razão comumente usada para explicar este resultado é a existência de restrição de crédito, pois neste caso o consumidor não consegue suavizar seu consumo. Em outras palavras, choques que afetam a renda tem grande impacto sobre o consumo. Finalmente, não há evidência de que as mudanças (previsíveis) do gasto do governo acarretam mudanças no consumo privado. Portanto, o consumo e o gasto do governo não são substitutos nem tão pouco complementares. Naturalmente, pode haver um efeito indireto no curto prazo se o gasto do governo impactar a renda corrente.

\section{BIBLIOGRAFIA}

Angeletos, G. M., Laibson, D., Repetto, A., Tobacman, J., \& Weinberg, S. (2001). The hyperbolic consumption model: Calibration, simulation, and empirical evaluation. Journal of Economic Perspectives, 15:47-68.

Araújo, E. A. J. (2006). Avaliação e teste de dois modelos de formação de preço de ativos baseados no consumo para o Brasil: Uma abordagem baseada em teoria da informação. Brazilian Business Review, 3:1-14.

Brady, R. (2008). Structural breaks and consumer credit: Is consumption smoothing finally a reality? Journal of Macroeconomics, 30:1246-1268.

Bugarin, M., Ellery Jr., R., Gomes, V., \& Teixeira, A. (2007). The brazilian depression in the 1980s and 1990s. In Kehoe, T. \& Prescott, E., editor, Great Depressions of the Twentieth Century, pages 287-304. Federal reserve Bank of Minneapolis, Minnesota.

Campbell, J. Y. \& Mankiw, G. N. (1989). Consumption, income and interest rates: Reinterpreting the time series evidence. In Blanchard, O. J. \& Fischer, S., editors, NBER Macroeconomics Annual, pages 185-214. MIT Press, Cambridge, MA.

Campbell, J. Y. \& Mankiw, N. G. (1990). Permanent income, current income, and consumption. Journal of Business and Economic Statistics, 8:265-280. 
Carrol, C. (1997). Buffer-stock saving and the life cycle/permanent income hypothesis. Quarterly Journal of Ecnomics, 112:1-55.

Cavalcanti, C. (1993). Intertemporal substituion in consumption: An empirical investigation for Brazil. Brazilian Review of Econometrics, 13:203-229.

Dickey, D. A. \& Fuller, W. A. (1979). Distribution of the estimators for auto-regressive time series with a unit root. Journal of the American Statistical Association, 74:427-431.

Ellery, R. \& Teixeira, A. (2012). O milagre, a estagnação e a retomada do crescimento: As lições da economia braseira nas últimas décadas. In Veloso, F., Ferreira, P. C., Giambiagi, F., \& Pesseditors, Desenvolvimento econômico: Uma perspectiva brasileira, pages 263-284. Elsevier, Rio de Janeiro.

Ellery Jr., R., Gomes, V., \& Sachsida, A. (2002). Business cycle fluctuations in Brazil. Revista Brasileira de Economia, 56:269-308.

Elliott, G., Rothenberg, T., \& Stock, J. (1996). Efficient tests for an autoregressive unit root. Econometrica, 64:813-836.

Engelhardt, G. V. (1996). Consumption, down payments and liquidity constraints. Journal of Money, Credit and Banking, 28:255-271.

Flavin, M. (1981). The adjustment of consumption to changing expectations about future income. Journal of Political Economy, 89:974-1009.

Flavin, M. (1985). Excess sensitivity of consumption to current income: Liquidity constraints or myopia? Canadian Journal of Economics, 18:117-136.

Friedman, M. A. (1957). Theory of the consumption function. Princeton, N.J., Princeton University Press.

Garcia, R., Lusardi, A., \& Ng, S. (1997). Excess sensitivity and asymmetries in consumption: An empirical investigation. Journal of Money, Credit and Banking, 29:157-176.

Gomes, F. (2004). Consumo no Brasil: Teoria da renda permanente, formação de hábito e restrição à liquidez. Revista Brasileira de Economia, 58:381-402.

Gomes, F. (2010). Consumo no Brasil: Comportamento otimizador, restricão de crédito ou miopia? Revista Brasileira de Economia, 64:261-275.

Gomes, F. (2013). Evolução do consumo de duráveis e não duráveis: Existe ajustamento lento no caso brasileiro? No prelo, Economia Aplicada.

Gomes, F., Issler, J., \& Salvato, M. (2005). Principais características do consumo de duráveis no Brasil e testes de separabilidade entre duráveis e não duráveis. Revista Brasileira de Economia, 59:33-60.

Gomes, F. \& Paz, L. (2004). Especificações para a função consumo: testes para países da América do Sul. 34:39-55, Pesquisa e Planejamento Econômico.

Gomes, F. \& Paz, L. (2010). Consumption in South America: Myopia or liquidity constraints? Economia Aplicada, 14:129-145.

Gourinchas, P. \& Parker, J. (2001). The empirical importance of precautionary saving. American Economic Review, 91:406-412.

Hall, R. (1978). Stochastic implications of the life cycle-permanent income hypothesis: Theory and evidence. Journal of Political Economy, 86:971-987. 
Hamilton, J. D. (1994). Time series analysis. Princeton, New Jersey, Princeton University Press.

Issler, J. \& Piqueira, N. (2000). Estimating relative risk aversion, the discount rate, and the intertemporal elasticity of substitution in consumption for Brazil using three types of utility function. Brazilian Review of Econometrics, 20:200-238.

Issler, J. \& Rocha, F. (2000). Consumo, restrição a liquidez e bem-estar no Brasil. Economia Aplicada, 4:637-665.

Karras, G. (1994). Government spending and private consumption: Some international evidence. Journal of Money, Credit, and Banking, 26:9-22.

Karras, G. \& Evans, P. (1996). Private and government consumption with liquidity constraints. Journal of International Money and Finance, 15:255-266.

Kwiatkowski, D., Phillips, P., Schmidt, P., \& Shin, Y. (1992). Testing the null hypothesis of stationary against the alternative of a unit root. Journal of Econometrics, 54:159-178.

Madsen, J. \& McAleer, M. (2000). Direct tests of the permanent income hypothesis under uncertainty, inflationary expectations and liquidity constraints. Journal of Macroeconomics, 22:229-252.

Newey, W. \& West, K. (1987). A simple positive semi-definite, heteroskedasticity and autocorrelation consistent covariance matrix. Econometrica, 55:703-708.

Phillips, P. C. B. \& Perron, P. (1988). Testing for a unit root in time series regression. Biometrika, 75:335346.

Reis, E., Issler, J. V., Blanco, F., \& Carvalho, L. (1998). Renda permanente e poupança precaucional: evidências empíricas para o Brasil no passado recente. Pesquisa e Planejamento Econômico, 28:233272 .

Sarno, L. \& Taylor, M. (1998). Real interest rates, liquidity constraints and financial deregulation: Private consumption behavior in the uk. Journal of Macroeconomics, 20:221-242.

Vaidyanathan, G. (1993). Consumption, liquidity constraints and economic development. Journal of Macroeconomics, 15:591-610.

Weber, C. E. (2002). Intertemporal non-separability and "rule of thumb" consumption. Journal of Monetary Economics, 49:293-308. 